\title{
Entirely irrelevant distractors can capture and captivate attention
}

\author{
Sophie Forster • Nilli Lavie
}

Published online: 12 October 2011

(C) The Author(s) 2011. This article is published with open access at Springerlink.com

\begin{abstract}
The question of whether a stimulus onset may capture attention when it is entirely irrelevant to the task and even in the absence of any attentional settings for abrupt onset or any dynamic changes has been highly controversial. In the present study, we designed a novel irrelevant capture task to address this question. Participants engaged in a continuous task making sequential forced choice (letter or digit) responses to each item in an alphanumeric matrix that remained on screen throughout many responses. This task therefore involved no attentional settings for onset or indeed any dynamic changes, yet the brief onset of an entirely irrelevant distractor (a cartoon picture) resulted in significant slowing of the two (Experiment 1) or three (Experiment 2) responses immediately following distractor appearance These findings provide a clear demonstration of attention being captured and captivated by a distractor that is entirely irrelevant to any attentional settings of the task.
\end{abstract}

Keywords Attentional capture · Attention - Attentional settings $\cdot$ Irrelevant distractors

In daily life, distraction often occurs from stimuli that appear to be entirely irrelevant to the current task-for example, the sight of a colleague entering the room may

\section{S. Forster $(\bowtie)$}

Department of Psychology and Helen Wills Neuroscience Institute, Tolman Hall, Berkeley,

University of California, Berkeley,

Berkeley, CA 94720-1650, USA

e-mail: sophie.forster@gmail.com

N. Lavie

Institute of Cognitive Neuroscience, University College London,

London, England, UK distract you from reading this article and capture your attention instead. Attentional capture research, however, remains highly contentious as to whether any type of stimulus can capture attention even when it bears no relevance to any task-related attentional settings. A considerable body of research has highlighted abrupt onsets as a particularly strong candidate for the type of stimuli that could cause attentional capture under such circumstances (see Yantis, 2000, for review). Abrupt stimulus onset indicates an environmental change and, due to the sociobiological importance of detection of such changes (e.g., in cases in which these may indicate a predator approaching), one might expect that the abrupt onset of a stimulus would capture attention and interrupt task performance even when the onset stimulus is entirely irrelevant to the task at hand.

We have previously (Forster \& Lavie, 2008a, b) established a measure of attentional capture from distractors designed, like many daily-life distractors, to be entirely irrelevant to the task performed. Participants performed a letter search around fixation. On a minority of trials, a distractor picture (of a famous cartoon character, e.g., superman) was presented in the periphery. These distractors were clearly irrelevant to the letter search in terms of shape and color (the distractors were colorful figures, whereas the search letters were gray), location (they never occupied nor predicted any of the target positions), and identity, yet produced robust interference effects on the search task performance. This result contrasts with previous attentional capture paradigms (e.g., Jonides \& Yantis, 1988; Theeuwes, 1992), in which the distractor appears in one of the letter search locations and is thus directly relevant to the search task. The interference effect did not depend on any singleton detection search strategy (cf. Bacon \& Egeth, 1994); it 
persisted even when the search target was not a singleton (rather it appeared among two other similar letters). When the search load was increased, however, it was eliminated, demonstrating that the interference was due to misallocation of attentional resources (see Lavie's load framework, e.g. for review, Lavie, 2010).

One potentially important feature of our irrelevant distractors was their abrupt onset. The distractor stimuli appeared in a peripheral location that was not occupied by any stimulus on the previous trial. Although their abrupt onset may have facilitated capture of attention, it also may have raised the possibility that in some sense the distractor stimuli were, in fact, relevant to general attentional settings for onset, since these signaled the start of each trial in the task (see Gibson \& Kelsey, 1998).

This alternative account has critical implications for the debate as to the existence of attentional capture by truly irrelevant stimuli. The strongest evidence for irrelevant capture so far has come from studies involving abrupt onset, but almost all previous studies purporting to demonstrate irrelevant attentional capture by onsets have also used discrete trial-by-trial stimulus display onsets. A few studies assessed capture by onset in tasks in which the target displays did not involve onset, either because target stimuli appeared as offsets (e.g., Jonides \& Yantis, 1988, or more recently, Schreij, Owens, \& Theeuwes, 2008), or because the start of a trial was signaled by the movement of a continuously present target (Kim \& Hopfinger, 2010). In such cases, onset is no longer part of task settings. Nevertheless, these studies have all used a form of dynamic event to signal the start of each trial and thus are not immune to the possibility that attentional capture by onset depends on general task settings for any form of dynamic event. Note that general task settings for dynamic changes may be a necessary but insufficient condition for capture by another change (e.g., a luminance change to an existing object, see Kim \& Hopfinger, 2010). In other words, such relevance may remain a necessary condition for capture by a new object onset even if insufficient for capture by other changes.

Another creative approach for avoiding Gibson and Kelsey's (1998) criticism is the "surprise capture" paradigm (e.g., Horstmann, 2002, 2005; Horstmann \& Becker, 2011), which demonstrates facilitation effects from the first occurrence of an unexpected color change either to the target, or to a background patch surrounding the target. This paradigm was adapted by Godjin and Kramer (2008) to demonstrate oculomotor capture from the first appearance of an abrupt onset distractor. An unexpected stimulus cannot be used as any form of task-relevant signal and therefore would seem irrelevant to any top-down attentional setting. However, these studies also involved general settings for dynamic changes (through either display onsets or color change on each trial). Therefore, it remains unclear whether surprise capture would occur also for unexpected stimulus changes in a task that involves neither onset nor any other form of dynamic changes as part of the task. In sum, all previous demonstrations of irrelevant attentional capture by abrupt onset could be alternatively explained by relevance to the attentional task settings, and critically the involvement of dynamic changes in the task (see also Burnham, 2007).

In the present study, we thus sought to examine whether attentional capture by irrelevant onset distractors can be found in a continuous task under conditions that rule out any attentional settings to onset. Participants worked through a matrix display (Fig. 1) from left to right (starting at the top), making sequential forced choice responses as to whether each item was a digit or a letter. During $30 \%$ of the matrices, a task-irrelevant cartoon character distractor was presented in the periphery at any time during the matrix performance except during the first or last response (Experiment 1) or first 10 or last two responses (Experiment 2). The matrix display remained on screen until a response had been made to the last matrix item. Because the stimulus display remained unchanged throughout the response sequence, participants had no reason to adopt attentional settings for abrupt onsets, or for any dynamic change. Given that the colorful distractor cartoon image was also irrelevant to the task concerning gray digits or letters and appeared in an irrelevant peripheral location outside the matrix, any interference effects produced by this irrelevant distractor would appear to reflect truly task-irrelevant attentional capture.

\section{Experiment 1}

Method

Participants Eight participants (two males of the ages 2234 years, $M=26$ ) participated in Experiment 1 .

Stimuli and procedure The experiment was created and run using E-prime.

Stimuli were presented on a PC with a 15 -in. monitor at a viewing distance of $60 \mathrm{~cm}$ (maintained using a chinrest). Each of the matrix items was equally likely to be either a digit or a letter (chosen at random from 1-9 or X, R, T, J, L, P, N, F, B), with the constraint that no more than two letters or digits could appear next to each other. The square matrix area subtended $4.8^{\circ}$ by $4.1^{\circ}$, with $1.4^{\circ}$ between adjacent items. Although selfpaced, participants were instructed to respond as fast as 
a

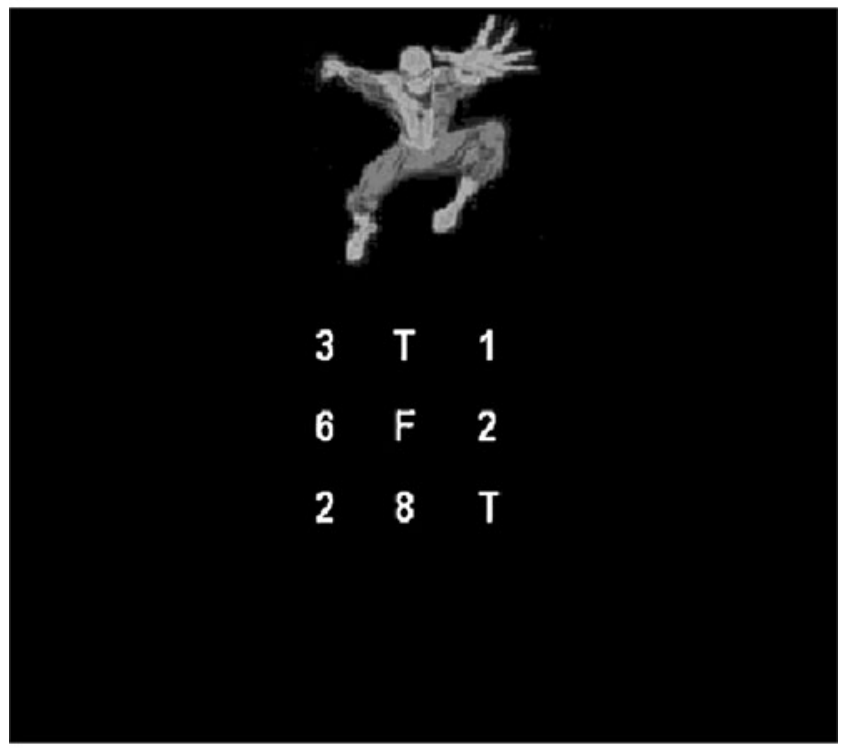

b

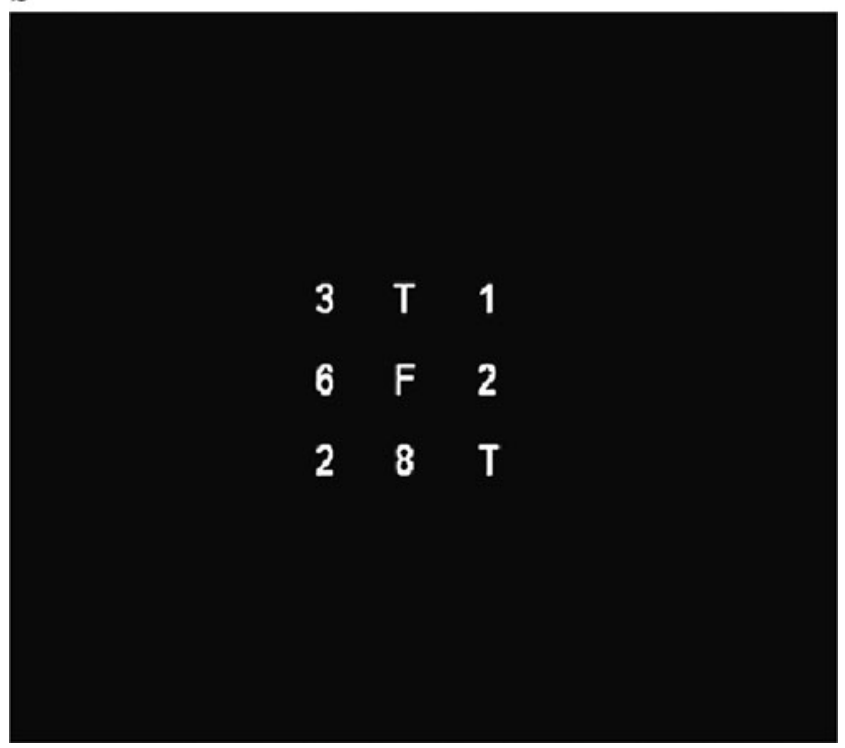

Fig. 1 Sample stimulus display with a an irrelevant distractor present and $\mathbf{b}$ no distractor. Participants were instructed to work through the matrix from left to right, starting at the top, and to indicate whether each character was a letter (pressing the " 0 " key) or a digit (pressing the " 2 " key). The distractor was presented immediately following one of the target responses. If, for example, the distractor was presented following the response to the third item ("1") in the matrix shown, then lag 0 refers to RT to the next item ("6"), lag 1 to RT to item "F," and so forth

possible while being accurate and to focus attention only on the letter/digit stimuli. A 90 -ms beep sounded immediately after incorrect responses.

A new matrix appeared immediately upon response to the last item in each matrix. Participants performed two matrices for practice, followed by eight blocks of 20 matrices. During six of the 20 matrices in each block, an irrelevant distractor (color image of one of six cartoon characters: Spiderman, Superman, Spongebob, Mickey Mouse, Donald Duck, and Pikachu), subtending $2.8^{\circ}$ to $4^{\circ}$ vertically by 2.8 to $3.2^{\circ}$ horizontally, was presented for $100 \mathrm{~ms}$ with equal likelihood either above or below the matrix (at least $1.5^{\circ}$ between the nearest edges of distractor and matrix), immediately following any but the first or last of the target responses. The combinations of distractor identity and position were fully counterbalanced between blocks.

\section{Results and discussion}

RTs over 2,000 ms were excluded from the analysis of both experiments reported. To assess attentional capture and any lasting effects that may reflect attention holding, mean RTs were calculated as a function of distractor condition and lag between the distractor presentation and the response target. Lag 0 refers to the RTs to the target of the first response following distractor presentation, and lag 1 refers to RTs to targets in the subsequent matrix position, and so on (see Fig. 1 for an example). Lag 0 included matrix positions $2-8$ (recall that the distractor was never presented during responses to the first or ninth item), lag 1 included 3-9, and so forth. To control for effects of matrix item position, the mean RTs in the distractor-present matrices at each lag were compared with the mean RTs to the equivalent range of matrix target positions in the matrices with no distractor present. An ANOVA with the factors of distractor presence (present, absent) and lag (0-4, see Note 1$)$ revealed a main effect of distractor presence, $F(1,7)=11.77, M S E=$ $1,762.00, p=.011, \eta_{p}{ }^{2}=.63$; and lag, $F(4,28)=6.19$, $M S E=2,428.04, p=.001, \eta_{p}{ }^{2}=.47$, which followed a linear trend, $F(1,7)=19.17, M S E=2,622.47, p=.003$, $\eta_{p}{ }^{2}=.73$. As can be seen in Fig. 2a, RTs were slower with distractor presence and at the earlier distractor lag positions. This was qualified by a significant distractor $\mathrm{x}$ lag interaction, $F(4,28)=2.92, M S E=1,192.47, p=.039$, $\eta_{p}{ }^{2}=.29$, which followed a linear trend, $F(1,7)=13.15, p=$ $.008, M S E=1,315.77, \eta_{p}{ }^{2}=.65$, reflecting reduced distractor interference with longer lags. There was significant interference at lag $0[M=60 \mathrm{~ms}, t(7)=3.26$, SEM $=$ $18.25, p=.014, d=2.46]$, and at lag $1(M$ distractor cost $=$ $82 \mathrm{~ms}), t(7)=2.89, S E M=28.45, p=.023, d=2.18$. There was a nonsignificant trend toward interference at lag $2, t(7)=$ 1.84, SEM $=13.78, p=.108, d=1.39$, but no further interference beyond these lags (all $t$ values $<1$ ). As can be seen in Fig. $2 b$, there were no effects of irrelevant distractor presence on the error rates, (all $F_{\mathrm{S}}<1$, see Note 2). These results demonstrate clear interference from abrupt onset distractors that were entirely irrelevant to a task that did not involve abrupt onsets of the stimulus display. The interfer- 

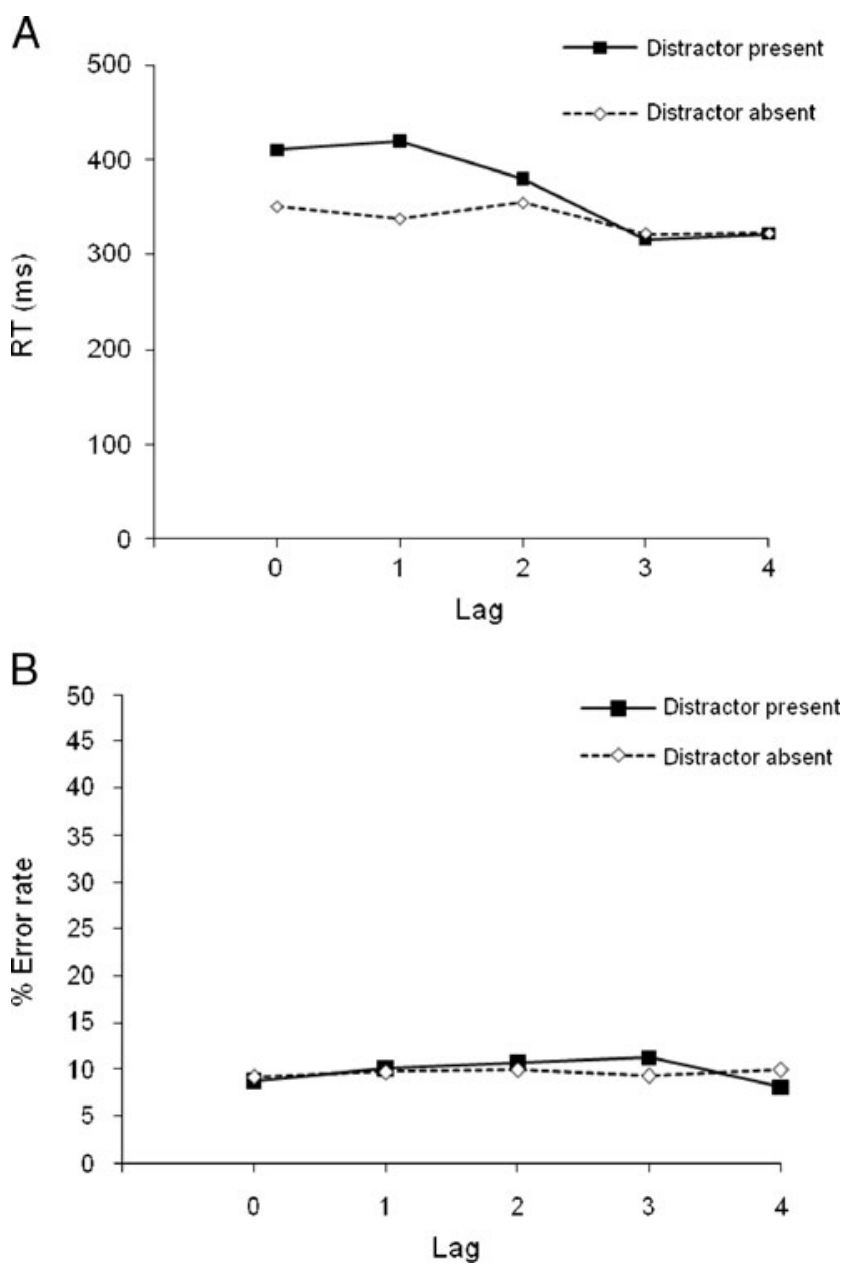

Fig. 2 Mean RT (a) and percentage error rate (b) as a function of irrelevant distractor presence (vs. absence) and lag in Experiment 1. Each lag represents a different range of item positions within the matrix (matched between the distractor and no distractor response matrices): Lag 0 reflects matrix positions 2-8 (note that the target was never presented in position 1 or 9), lag 1 reflects matrix positions 3-9, lag 2 reflects matrix positions $4-9$, lag 3 reflects matrix positions 5-9, lag 4 reflects matrix positions $6-9$, lag 5 reflects matrix positions $7-9$

ence effects lasted over two to three responses, suggesting that the distractor captured and captivated attention.

\section{Experiment 2}

In Experiment 1, there were only nine targets per matrix. In Experiment 2, we increased the number of matrix items to 36 , and the irrelevant distractor never appeared during the first 10 target responses. In this way, the matrix onset was even further removed from the distractor presentation. In addition, in Experiment 1, error feedback was given with a tone. Although the low error rate would have meant that tone feedback was infrequent, given that the tone had an onset, we deemed it important to avoid any onset settings related to the tone. In Experiment 2, therefore, feedback tones were not given during the task (only during practice).

Method

Participants Ten new participants (four males) between the ages of 19 and $27(M=23)$ participated in Experiment 2.

Stimuli and procedure All stimuli and procedure were the same as in Experiment 1, with the following exceptions. Each matrix consisted of three rows of 12 items each (chosen at random from Experiment 1's set, with the constraint of no more than six consecutive letters or numbers. Each row subtended $21.1^{\circ}$ horizontally. The irrelevant distractor never appeared during the responses to the first 10 target items in each matrix, nor to the final two items in each matrix, but appeared with equal likelihood during responses to any of the remaining 24 items. Participants were instructed that in the event of losing their place in the matrix, they should press the spacebar and then continue making responses, even if they were not sure, until a new sequence appeared.

Results and discussion

As in Experiment 1, RTs at each lag were compared to RTs on the matching no distractor matrix positions in a $2 \times 15$ ANOVA, with the factors of distractor presence (present, absent) and lag (0-14). A main effect of lag was found, $F(14,126)=2.45, M S E=1384.34, p=.001, \eta_{p}{ }^{2}=.214$, with a linear trend, $F(1,9)=10.09, p=.011, M S E=$ 1725.16, $\eta_{p}^{2}=.53$, reflecting slower RTs for lags associated with earlier matrix positions. The main effect of distractor presence in the matrix was not significant, $F<1$; however, importantly, there was an interaction between lag and distractor presence, $F(14,126)=2.58$, $M S E=1310.50, p=.003, \eta_{p}{ }^{2}=.22$, once again showing a linear trend, $F(1,9)=20.25, p=.001, M S E=1722.83$, $\eta_{p}{ }^{2}=.69$, reflecting distractor interference only on the responses immediately following distractor presentation.

As can be seen in Fig. 3, mean RT was significantly longer $(M$ cost $=58 \mathrm{~ms})$ in the presence of the irrelevant distractor (lag 0) compared to in the equivalent matrix positions (11-34) on no distractor displays, $t(9)=2.47$, $S E M=23.46, p=.035, d=1.65$. Significant distractor interference $(M=49 \mathrm{~ms})$ continued at lag 1 as compared with RTs to no distractor targets in the equivalent positions (12-35), $t(9)=6.16, S E M=7.99, p<.001, d=4.11$, twotailed. As in Experiment 1, the magnitude of the interference effect at lag 1 did not significantly differ from that at lag $0, t<1$. As can be seen in Fig. 3a, significant interference $(M=32 \mathrm{~ms})$ remained at lag $2, t(9)=2.45$, $S E M=13.19, p=.037, d=1.63$, two-tailed, but was 

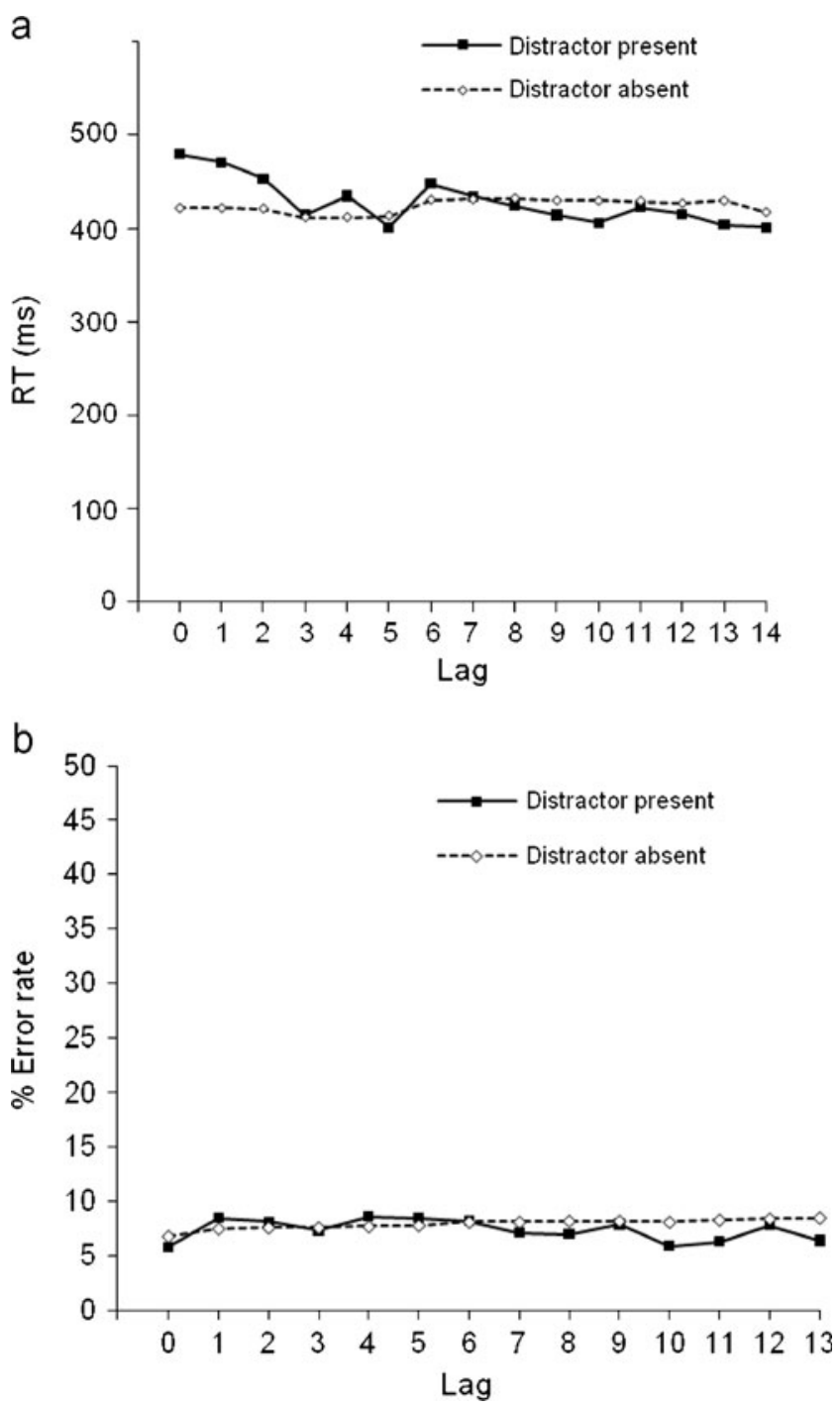

Fig. 3 Mean RT (a) and percentage error rate (b) as a function of irrelevant distractor presence (vs. absence) and lag in Experiment 2. As in Experiment 1, the no distractor baseline reflects the set of no distractor matrix positions that match those distractor matrix positions associated with each lag

reduced to nonsignificance by lag $3(t<1$, for all subsequent lags, $p>.25$ with the exception of a trend toward interference at lag $4, M=23 \mathrm{~ms}, t(9)=2.18, S E M=$ $16.34, p=.057, d=1.45)$. Thus, as in Experiment 1, the abrupt onset distractor produced significant interference effects that lasted over several target responses, suggesting it captured and captivated attention during performance of a task that involved no attentional settings for onset.

A between-experiments ANOVA confirmed that increasing the display size from nine (Experiment 1) to 36 (Experiment 2) and removing the feedback tones had no effect on the magnitude of distractor effects obtained on either the current target response or at lags 1 and $2(F<1$ for both the main effect of experiment and for the interaction between experiment and lag).
There were no significant effects on percentage error rates, for the comparison of current target response and no distractor (all $p \mathrm{~s}>.097$, Fig. 3b). An additional analysis revealed that participants reported "losing their place" (by pressing the spacebar) during a mean of $7 \%$ of matrices and were no more likely to do so during a display on which an irrelevant distractor was presented, $t<1$.

\section{General discussion}

The results of the present study clearly demonstrate interference from distractors that are entirely irrelevant to the task at hand and to any attentional settings associated with task performance. The prolonged cost on the letter search RT produced by the presence of an irrelevant singleton distractor in the periphery suggests that the irrelevant distractor captured and captivated attention. The fact that the distractor cost was found despite its being entirely irrelevant to the task in terms of color, shape, location, and identity, as well as any general attentional settings for task display onset, or any other dynamic changes, is highly suggestive of attentional capture that is truly not contingent on the attentional settings in the task.

Note that the present paradigm not only satisfies Gibson and Kelsey's (1998) requirement for the "strongest test" of attentional capture by abrupt onset (having an unchanging target display), but also meets the three criteria recently outlined by Burnham, Neely, Naginsky, and Thomas (2010) for demonstrating irrelevant attentional capture: The target was not a singleton; rather, each of the matrix items were response targets, and they all shared the same visual features. The distractor did not predict the target location, and the task did not induce any "display-wide" attentional settings related to any distractor feature (e.g., neither in terms of display onset or offset nor in terms of other cues that signal the start of each display, such as a color change). The cost to task performance in the presence of such an irrelevant distractor therefore may reflect truly irrelevant attentional capture.

To our knowledge, the only previous study directly investigating the issue of attentional capture by abrupt onsets, within a paradigm designed to preclude attentional settings for any dynamic changes to the stimulus display (Franconeri, Simons, \& Junge, 2004), examined facilitation effects rather than interference effects. In their study, Franconeri et al. monitored eye movements during a letter search task and changed the stimuli while participants made large saccades away from the display. Results showed shallower search slopes when nonpredictive, abrupt onset spots appeared around the target letter (as compared with when they surrounded a nontarget letter, or were absent), suggesting task-irrelevant attentional capture. However, 
although the participants did not see the onset of each stimulus display, they would have been anticipating new objects (i.e., the new target stimuli) in the display and, therefore, the abrupt onset spots, appearing as additional new objects, may have been prioritized on the basis of their relevance to attentional settings for new objects. The present study avoided this issue, having a continuous display presentation, and also extended Franconeri et al.'s findings by demonstrating that abrupt onsets can capture attention even when presented as entirely task-irrelevant distractors.

We note that the present paradigm does not allow for demonstration of the spatial capture of attention (cf. Folk \& Remington, 1998). Indeed, it is very difficult to conceive of a paradigm sensitive to show spatial effects that would not intrinsically make the distractor location task-relevant. However, we have previously established (Forster \& Lavie, 2008a) that interference from these task-irrelevant distractors was eliminated when attentional capacity was exhausted under high perceptual load in the search task. These results demonstrate that the distractor effect clearly depends on the allocation of attentional resources to the irrelevant distractor: In other words, that it captured attention. In addition, the prolonged duration of distractor interference effects is highly suggestive of not only capture but also captivation of attention by the irrelevant distractors.

The lasting effects of our distractor on performance may have been due to the strong contrast between the monotonous nature of a continuous task as compared with the capturing nature of an abrupt onset. In addition, although the distractor processing was initiated despite the distractors' irrelevance to any top-down task goals (these concerned letter vs. digit discrimination), our use of semantically meaningful distractors may have encouraged higher level processing of distractors, and that may have contributed to their captivating effect.

The present study therefore establishes that an abrupt onset distractor can capture and captivate attention despite its true irrelevance to the current task and any related attentional settings. The experimental paradigm presented here could be used in future research to further characterize such attentional capture. For example, interesting new avenues would be identifying the necessary properties that an abrupt onset must have in order to cause attentional capture: Our study has shown irrelevant attentional capture by an infrequent, meaningful, and full-color abrupt onset distractor; future research should clarify whether abrupt onsets distractors can also capture attention in the absence of attentional settings when they are frequent, meaningless, or of lower visual salience than the target stimuli.

Indeed, a previous study using the inattentional blindness paradigm (Jingling \& Yeh, 2007) has shown that people may fail to notice the onset of a very small stimulus (a white line subtending $0.04^{\circ}$ by $0.003^{\circ}$ ) while engaging in a change detection task concerning much larger and colorful stimuli (a disk subtending $7.4^{\circ}$ by $6.3^{\circ}$ ) unless the task involves attentional settings for onset (each display being preceded with a blank screen). This result indirectly suggests that an entirely task-irrelevant abrupt onset stimulus needs to exceed a threshold level of salience on other dimensions (such as size) in order to capture attention. However, the inattentional blindness paradigm involves additional factors that may influence sensitivity to detect attentional capture (e.g., the stimulus on the last trial is unexpected, and detection is self-reported in a surprise question following the task response, thus confounding memory failures). A more direct test using online measures of attentional capture, as used here, can better address this question.

Of course, the present results do not rule out that attentional capture effects are enhanced when participants do adopt attentional settings for an abrupt onset. However, contrary to previous claims, the present results clearly demonstrate that such an attentional setting is not a necessary condition for attentional capture.

Author Note This research was supported by a Wellcome Trust award (WT080568MA) to N. L.

Open Access This article is distributed under the terms of the Creative Commons Attribution Noncommercial License which permits any noncommercial use, distribution, and reproduction in any medium, provided the original author(s) and source are credited.

\section{References}

Bacon, W. F., \& Egeth, H. E. (1994). Overriding stimulus-driven attentional capture. Perception \& Psychophysics, 55, 485-496.

Burnham, B. R. (2007). Displaywide visual features associated with a search display's appearance can mediate attentional capture. Psychonomic Bulletin \& Review, 14, 392-422.

Burnham, B.R., Neely, J.H., Naginsky, Y., \& Thomas, M. (2010). Stimulus-driven attentional capture by a static discontinuity between perceptual groups. Journal of Experimental Psychology: Human Perception \& Performance, 36(2), 317-329.

Folk, C. L., \& Remington, R. (1998). Selectivity in distraction by irrelevant featural singletons: Evidence for two forms of attentional capture. Journal of Experimental Psychology. Human Perception and Performance, 24, 847-858.

Forster, S., \& Lavie, N. (2008a). Attentional capture by entirely irrelevant distractors. Visual Cognition, 16, 200-214.

Forster, S., \& Lavie, N. (2008b). Failures to ignore entirely irrelevant distractors: The role of load. Journal of Experimental Psychology. Applied, 14, 73-83.

Franconeri, S. L., Simons, D. J., \& Junge, J. A. (2004). Searching for stimulus-driven shifts of attention. Psychonomic Bulletin \& Review, 11, 876-881.

Gibson, B. S., \& Kelsey, E. M. (1998). Stimulus-driven attentional capture is contingent on attentional set for displaywide visual features. Journal of Experimental Psychology. Human Perception and Performance, 24, 699-706.

Godjin, R., \& Kramer, A. F. (2008). Oculomotor capture by surprising onsets. Visual Cognition, 16, 279-289.

Horstmann, G. (2002). Evidence for attentional capture by a surprising color singleton in visual search. Psychological Science, 13, 499-505. 
Horstmann, G. (2005). Attentional capture by an unannounced color singleton depends on expectation discrepancy. Journal of Experimental Psychology. Human Perception and Performance, 31, 1039-1060.

Horstmann, G., \& Becker, S.I. (2011). Evidence for goal-independent attentional capture from validity effects with unexpected novel color cues-A response to Burnham (2007). Psychonomic Bulletin \& Review, 18(3), 512-517.

Jingling, L., \& Yeh, S. (2007). New objects do not capture attention without a top-down setting: Evidence from an inattentional blindness task. Visual Cognition, 15, 661-684.

Jonides, J., \& Yantis, S. (1988). Uniqueness of abrupt visual onset in capturing attention. Perception \& Psychophysics, 43, 346-354.

Kim, S., \& Hopfinger, J. B. (2010). Neural basis of visual distraction. Journal of Cognitive Neuroscience, 22, 1794-1807.

Lavie, N. (2010). Attention, distraction and cognitive control under load. Current Directions in Psychological Science, 19, 143-148.

Schreij, D., Owens, C., \& Theeuwes, J. (2008). Abrupt onsets capture attention independent of top-down control settings. Perception \& Psychophysics, 70, 208-218.

Theeuwes, J. (1992). Perceptual selectivity for color and form. Perception \& Psychophysics, 51, 599-606.
Yantis, S. (2000). Goal-directed and stimulus-driven determinants of attentional control. In S. Monsell \& J. Driver (Eds.), Attention and Performance (Vol. 18, pp. 73-103). Cambridge, MA: MIT Press.

\section{Notes}

1. Later lags included fewer trials; we therefore restricted our analysis to lags involving at least 24 trials (up to lag 4 in Experiment 1 and lag 14 in Experiment 2).

2. We note that like in many studies using RT as the main measure, the manifestation of attentional capture on RT rather than error rate is common also in the attentional capture literature, although some previous studies have found effects on both RT and error rate (e.g., Kim \& Hopfinger 2010). The self-paced presentation method in our study seems likely to have made our paradigm less sensitive to attentional capture effects on errors, since participants had time to simply recover from presentation of the distractors and respond correctly, albeit more slowly, to the target. 